\title{
LAS REDES SOCIALES DE LA FAMILIA DEVESA HERRERA EN LA COMUNIDAD MARINERA CASTILLO-PERCHÉ. ESTUDIO DE CASO
}

Social Networks Devesa Herrera Family Seaside Community Castillo-Perché. Case Study

\section{Cynthia Acevedo Rodríguez Nereyda Moya Padilla}

Resumen: El estudio de las redes sociales de familia en los entornos marineros tiene una gran significación para las ciencias sociales en la contemporaneidad, el trabajo que se presenta parte de establecer el origen y genealogía de la familia Devesa Herrera, su ubicación como red familiar en el contexto de la comunidad, para establecer el rol que desempeña como red familiar en la comunidad marinera Castillo-Perché. Desde una visión sociocultural se enfatiza en la tradición pesquera como elemento distintivo en torno al cual se establecen los nodos y vínculos que componen a esta red familiar, lo que permite ofrecer un análisis de las funciones sociales de esta familia, en un contexto mediado por la pesca como actividad económica fundamental en la comunidad.

Palabras claves: Redes sociales, familia Devesa Herrera y comunidad marinera. 
Abstract: The study of social networks of family sailors environments is of great significance for the social sciences in contemporary times, the work presents a part of the origin and genealogy of the family Devesa Herrera, location and family network in the context community, to establish the role it plays as a family network in the seafaring community Castillo-Perché. From a sociocultural view emphasizes the fishing tradition as a distinctive feature around which sets out the nodes and links that make up this family network, thereby offering an analysis of the social functions of this family, in mediated context fishing as a key economic activity in the community.

Keywords: Social networks, Devesa Herrera family and seafaring community.

\section{Introducción}

En la época contemporánea los estudios sobre la sociedad, las múltiples relaciones que se establecen en su interior y aquellas que se dan entre esta y la naturaleza han cobrado gran importancia.

En particular, el estudio sobre el funcionamiento y establecimiento de redes sociales tiene un lugar importante en el marco de las ciencias sociales, lo que justifica el número considerable de investigadores e instituciones que se dedican al tema "al estar orientados a descubrir la dinámica interna de las sociedades, su comportamiento y sus fortalezas, sobre la base del conocimiento del tipo de relaciones que las identifican, para enfrentar determinadas situaciones en un contexto y de su existencia ante la adversidad" (Miranda Vera, C. \& Castellanos González, M., 2007: 5). 
La preocupación por la realización de estos estudios está asociada al poderoso avance de las comunicaciones modernas, por las que el hombre comienza a tener la posibilidad real de ampliar y modificar su red de relaciones sociales, trascendiendo las barreras físicas espaciales, en períodos muy cortos de tiempo en el proceso de la comunicación interpersonal.

Es por ello que el estudio sobre redes sociales se convierte en una necesidad y una herramienta para el avance del conocimiento y su socialización. De ahí el propósito de esta investigación, orientado a sistematizar desde el punto de vista teórico, los elementos acerca de las redes sociales, específicamente en las familias al expresar los canales de comunicación tanto natural como institucional que permiten el movimiento del conocimiento tradicional y la información en un contexto comunitario, por lo que es de vital importancia su comprensión, al formar parte de un sistema de valores que posibilita establecer una visión más completa y acabada de la realidad social.

Para su realización fue necesario el empleo de la metodología cualitativa, fundamentalmente el método etnográfico, además del empleo de diversas técnicas como el análisis de documento, la entrevista a profundidad, la observación participante y la encuesta genealógica, por ser estos los fundamentos metodológicos que más se ajustan a los procesos socioculturales estudiados en el contexto natural, en este caso particular, de carácter familiar.

\section{Las redes sociales de familia. Definición y lugar en las estructuras sociales}

La teoría de redes se centra en la estructura social, en el soporte de vínculos que liga a los miembros individuales y colectivos de la sociedad. "Este enfoque remite básicamente a la coordinación social, es decir, se enmarca en las formas de tomar decisiones y de estructurar los conflictos en un determinado sistema de relaciones y vínculos como mecanismos de integración". (Albornoz \& Alfaraz, 2006: 17). 
El término de red social se utiliza para definir a:

Una estructura que cuenta con un patrón característico, o sea, donde diversos individuos mantienen distintos tipos de relaciones entre las que predominan las familiares, de amistad, de intercambios de conocimientos, costumbres y estilos de vida, relaciones de trabajo, originadas fundamentalmente a través de la convivencia y mediante la participación en las diferentes actividades socioculturales que se realizan en su contexto. (Merelo, 2004: 7).

Cada red social posee su propia estructura que da solidez a las relaciones que se establecen en ella para lograr un objetivo común, está sustentada a partir del intercambio de información mediante sus particulares canales de comunicación para efectuar determinadas interacciones y servicios conforme a sus necesidades e intereses, lo que le da un significado peculiar a su evolución y transformación en la medida que lo hace cada uno de sus integrantes.

De las redes sociales, emanan por tanto, las redes del conocimiento, redes en las que individuos, grupos y organizaciones, o también actores sociales en su conjunto; se interrelacionan en un intercambio dinámico para seleccionar, captar, apropiarse, difundir y crear conocimiento. Están conformadas por nodos y vínculos. El primero constituye ser el núcleo de sus relaciones, pues favorece la estabilidad y permanencia en su conformación; el segundo funciona como nexos o puentes que entrelazan a cada elemento de la estructura que conforma la red, manteniéndolos vinculados unos con otros.

En un contexto más social y dinámico, las redes sociales son las interacciones humanas en la producción, almacenamiento, distribución, transmisión, acceso y análisis de los conocimientos producidos por el hombre de manera sistemática o por el interés personal o grupal por compartir datos de cualquier índole y a través de cualquier medio, con el objeto de desarrollar sus capacidades de creación, entendimiento, poder, estudio y transformación de la realidad que lo rodea en un ámbito territorial y en un determinado contexto económico social. 
Las redes sociales operan en diversos niveles, desde las relaciones de parentesco hasta otros nexos, organizaciones y en los diferentes espacios en que despliegan su actividad.

Las redes sociales de familia por ejemplo están formadas por una cadena de relaciones que se producen en un período y espacio determinado, donde se analizan las problemáticas de la vida cotidiana y se intercambian estrategias y experiencias, cuya finalidad es el fortalecimiento de las mismas y lograr con ello, mejores resultados en la sociedad, permitiendo un trabajo conjunto desde su propio ámbito de acción.

Como expresión del alto nivel de complejidad que poseen, que es su propiedad principal, se pueden identificar cuatro mecanismos de integración que las caracterizan:

La negociación y la racionalidad deliberativa, que se desprenden de su particular modo de toma de decisiones; la función de traducción, que deriva de la importancia de los flujos de información y de la comunicación entre una membrecia [sic] heterogénea y diferenciada; y la confianza mutua entre los participantes, que en el contexto de las redes sociales como entidades complejas se expresa como un equilibrio inestable entre la confianż personal o normativa, la confianz̧a técnica y la confianza basada en el principio de la reciprocidad. (González \& Basaldúa, 2007: 6).

\section{Función social de las redes sociales de familia en el contexto de las comunidades marineras}

El trabajo familiar en redes puede ser apreciado en distintas situaciones, entre las cuales están aquellas presentes en el sector pesquero donde se desarrollan prácticas de producción en las que participan todos sus miembros, formando organizaciones dinámicas de la fuerza de trabajo. 
Estos estudios en una comunidad:

Se orientan a descubrir la dinámica interna que en ellas se presenta, así como su comportamiento, sus fortalezas, sobre la base del conocimiento del tipo de relaciones que la identifica, siendo este un criterio importante para determinar el capital social con que cuenta para enfrentar determinadas situaciones y sobre esa base realizar estrategias de trabajo con la comunidad. (Miranda \& Castellanos, 2008:8).

Por tanto, las redes sociales de familia tienen como función social en el contexto marinero la continua interacción e intercambio como expresión de la actividad humana al producir, almacenar y distribuir conocimiento científico o tradicional por medio de la transmisión oral. Buscan constantemente soluciones a los problemas que más les afectan, se ayudan mutuamente, comparten conocimientos, experiencias e ideas, desempeñándose como buenos actores sociales en beneficio de su comunidad, estableciendo relaciones de reciprocidad dentro de ellos como institución que son, al poseer cada miembro expectativas recíprocas, demostrando a su vez el rol y estatus que desempeñan.

\section{Antecedentes y orígenes de la familia Devesa Herrera}

La llegada del siglo XX para la sociedad cienfueguera significó la aparición de un nuevo contexto político y social, especialmente el flujo migratorio de origen hispano. Para nuestra investigación connotación esencial lo tiene el flujo de los canarios.

De este contexto son los ancestros paternales de la familia Devesa Herrera, que descendientes de familias pescadoras, inmigraron de España por la situación económica existente y se asentaron en la primera década del siglo XX en la comunidad marinera del Perché, específicamente en el centro sur de Cienfuegos, donde apenas existían ocho casitas de guano y madera en mal estado.

A su arribo transitaron por momentos difíciles, producto de la situación económica que existía en el país; un país con características 
neocoloniales, independientemente de que dicha comunidad se fue conformando sobre la base de una aparente prosperidad ligada a la construcción de "La Fortaleza Nuestra Señora de los Ángeles de Jagua."

Las entrevistas realizadas a Miguel Devesa Herrera (nieto) nos permite afirmar que aún con estas vicisitudes, al llegar y establecerse en la comunidad del Perché, Jaime Devesa (abuelo) logró mantener su "bienestar" tanto económico como social mediante la práctica de la pesca como actividad tecnoproductiva fundamental. Esto generó la conformación de una sólida red de familia que se desarrolla desde entonces.

La investigación nos permitió contrastar que al interior de la red familiar se observa una diversidad de profesiones, intereses, niveles culturales y de formación, pero en sentido general se aprecia un acercamiento sistemático a la práctica de la pesca no solo como actividad económica y profesional sino como una acción de cotidianidades que está marcando socioculturalmente la actividad intrafamiliar y constituye un punto medular de cohesión social e institucional que se sustenta en los saberes populares imprescindible para el desarrollo de las prácticas tecnoproductivas, siendo estas últimas las formas donde se centra la familia marinera y distinguen sus diversos espacios y contextos.

A continuación se muestra la representación gráfica de la familia Devesa Herrera, a partir de los resultados de la encuesta genealógica. La filiación que se traza a continuación es patrilineal porque exclusivamente se traza por vía masculina, o sea, el ego (designa el "yo" desde cuyo punto se consideran las relaciones de parentesco) sigue las líneas genealógicas ascendiente y descendiente solo a través de los varones dado el énfasis que se atribuye, dentro de la familia marinera, al ser el miembro cabecera de la misma. Obsérvese que esto no significa que los individuos emparentados por filiación sean solo varones; en cada generación hay parientes de ambos sexos. Sin 
embargo, en el paso de una generación a otra solo son pertinentes los lazos masculinos; los hijos de las mujeres se omiten en el cómputo de la filiación.

\author{
Gráfico No. ${ }^{\circ} 1$
}

Árbol genealógico de la familia Devesa

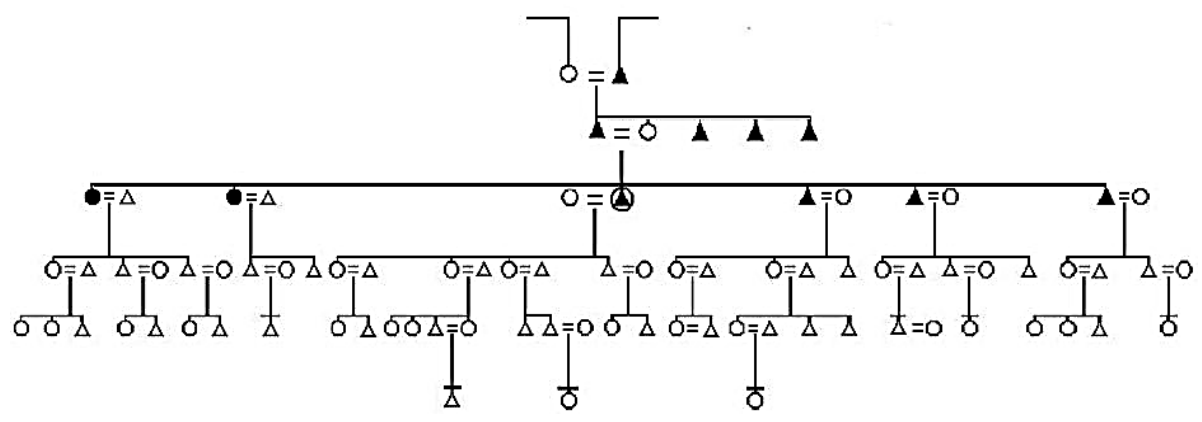
$\Delta \quad$ Varón
O Hembra
$=$ Casado con
Descendiente de
ワHermano de
(2) Ego cuya genealogia se muestra

\title{
EGO
}

Los antropólogos emplean la palabra ego para designar el "yo" desde cuyo punto de vista se consideran las relaciones de parentesco. A veces resulta necesario establecer si la persona de referencia es un ego varón o un ego mujer.

Fuente: Elaborado por la autora a partir de la aplicación de la encuesta genealógica.

\section{La familia Devesa Herrera como expresión de las redes sociales en la comunidad marinera Castillo-Perché}

Desde la perspectiva sociocultural, la pesca como actividad económica primordial de la red familiar Devesa Herrera, incide notablemente en la estructura social y en la organización fundamental del proceso de experiencias en la construcción de una subjetividad colectiva 
sustentada en la tradición pesquera condicionada histórica y socialmente por las características del contexto marino - costero.

Los miembros que conforman esta red familiar al vivir en una comunidad marinera presentan un profundo conocimiento acerca de la pesca y sus derivados, pero lo que les diferencia a uno del otro, es que cada miembro se especializa en diferentes aspectos en torno a la pesca. A continuación se hará alusión solamente a algunos ejemplos relacionado con lo antes planteado.

Miguel Devesa, miembro (Ego) de esta familia al desempeñarse como patrón de un barco, conoce mejor que sus demás hermanos sobre la distribución de los peces, según como esté la marejada y el tiempo, así como también se especializa en descifrar los nombres de estas especies marinas según su tipo. Eduardo Devesa, presenta mayor conocimiento en cuanto a la elaboración de las artes de pesca, en especial de la tarraya. Jesús Devesa tiene un mayor desenvolvimiento en cuanto a la reparación de los botes y chalanas; y si de avíos [sic] se trata, el que mejor domina esos conocimientos es Miguel Francisco.

Los nexos entre esta red de familia son muy fuertes. Todos mantienen excelentes relaciones entre sí y se apoyan unos a otros constantemente sin recibir nada a cambio. Son muy unidos, uno cuida del otro y viceversa. Cada miembro cumple una función social dentro de la misma en correspondencia con la concepción de su organización interna.

Expondremos a continuación la semejanza que existe entre los miembros de esta red familiar de acuerdo a los testimonios adquiridos durante la investigación, por medio de las entrevistas a sus miembros.

Miguel Devesa plantea:

Me llevo bien con todos. No estoy aquí peleado con nadie, ni con mi familia, amigos y ni vecinos. Siempre que nos vemos hablamos de pesca, intercambiamos ideas sobre cómo se debe hacer la tarraya, sobre negocios, 
en fin, todo relacionado al mar y a la pesca o sobre cualquier cosa que necesite de ellos que yo crea que me puedan ayudar...

Lázaro Luis Devesa afirmaba: "Con todos me llevo bien, ellos vienen y conversan... Tenemos el mismo trabajo, yo soy pescador y ellos también, vivimos en el mismo lugar y tenemos que llevarnos bien."

En las observaciones realizadas se pudo constatar que esta familia se distingue por la existencia de dos tipos de nodo. Por ejemplo: Uno es respecto a la actividad tecnoproductiva de la pesca y el otro relacionado a los días festivos del año. El primer nodo tiene un espacio de materialización en el muellecito creado por ellos mismos donde acostumbran a desembarcar cuando llegan de la larga y agotada jornada de trabajo. Es precisamente ahí donde Eduardo Devesa -líder principal-, en junto con los demás actores pone en marcha los nodos sociales del conocimiento, al ser portador del mismo; pues mayormente allí establecen sus conversaciones e intercambian experiencias, criterios y habilidades. Es así como transmiten sus conocimientos a los más pequeños de la casa y a todo vecino o visitante que se interese en adquirirlo.

El segundo nodo se desarrolla mayormente en las fechas conmemorativas. El encargado de organizarlas y dirigirlas con el apoyo y la cooperación de todos los demás miembros que conforman esta red familiar es Jesús Devesa. Ejemplo de estas celebraciones son los días de las madres, los cumpleaños, las navidades, fin de año, entre otros.

La familia en estudio se caracteriza por tener vínculos densos y cerrados, con un alto grado de conexión, donde cada individuo colabora al compartir en conjunto sus creencias, normas, valores, conocimientos, experiencias, cultura, estatus social y también porque los miembros tratan de encontrar la manera de propiciar soluciones a sus problemas. 
En toda comunidad marinera, prevalece la pesca como actividad económica fundamental y como medio de sustento de los miembros que la pueblan. La comunidad marinera Castillo-Perché es un ejemplo de lo antes planteado y muestra de lo mismo lo constituye la red de familia Devesa Herrera. Es por eso, que todas las actividades realizadas en el seno de una comunidad de esta naturaleza, giran en torno a la pesca.

Mediante las observaciones realizadas se pudo constatar la participación de los miembros de esta red familiar en todas las actividades que dirige y organiza la comunidad. Dígase las exposiciones de artes de pesca, concursos de elaborar comidas marineras; en las celebraciones populares y patronales, en las competencias deportivas incluyendo otros juegos. Del mismo modo colaboran en los trabajos políticos e ideológicos en el CDR, entre otros.

En las observaciones realizadas, también se pudo estimar el papel social que desempeñan los miembros de la red familiar Devesa Herrera mediante las interacciones que producen entre sí, siendo estas afectivas y de enseñanza, trasmitida a todos sus miembros.

A partir de ello se considera diversas interacciones socioculturales a lo interno de la red, las cuales:

Determinan los patrones de interacción social, es decir las maneras en que se reproducen los códigos que representan el sistema de significantes socialmente asumido por los diferentes colectivos y grupos humanos desde la praxis. Los tipos de relaciones se pueden manifestar en diferentes niveles en dependencia de su funcionalidad en la cotidianidad (individuoindividuo; individuo-grupo; individuo-comunidad). Abarcan diferentes aspectos: económicos, ideológicos, psicológicos, etc; desarrollados a partir de las normas de interacción social que determinan a partir de las redes de interacción los elementos más significativos que caracterizan a las redes. (Díaz, 2005: 4). 
Teniendo en cuenta este criterio se presentan a continuación los diversos tipos de relaciones expresados por la red social de la familia Devesa Herrera. De acuerdo con las observaciones realizadas dentro de la red de familia Devesa Herrera, se presenció en diferentes niveles de acuerdo a su tipo, funcionalidad familiar o social, en la actividad económica o intrafamiliar dentro de la cotidianidad; los tipos de relaciones manifestados por estos.

Es necesario dejar claro que esta relación se desarrolla dentro de dos procesos fundamentales: la producción y reproducción de los conocimientos populares, estableciéndose una relación individuo-individuo que se expresa mediante padres e hijos, madres e hijas, tíossobrinos, de forma oral, introduciendo frases y códigos en el lenguaje de acuerdo al espacio y contexto donde se encuentren, como se ha señalado en los epígrafes anteriores.

El segundo proceso se desarrolla en la relación individuo-grupo; en la familia este elemento se evidencia en la relación normativa y se establece durante la construcción de los avíos de pesca y la práctica de las diferentes artes de pesca. También se establece dicho proceso de relaciones durante las reuniones entre familiares, amigos o vecinos.

De acuerdo a lo antes abordado los Devesa Herrera establecen en el seno de su red familiar un grupo de interacciones que están mediadas por sus relaciones de poder y de roles. En el trabajo de campo se pudo constatar por medio de la observación y conversaciones informales que las que más sobresalen son mediante abuelo-nietos, padre, hijo, así como también tíos-sobrinos u otros miembros que la conforman.

Vale destacar que en esta interacción es donde se colectiviza el saber popular, donde se evidencia una vez más la transmisión de conocimientos de generación en generación, en especial, de las tecnologías como una de sus manifestaciones empleadas durante las artes de pesca. 
En las entrevistas y conversaciones informales se aprecia un sin número de prácticas socioculturales dirigidas en lo fundamental a la transmisión de saberes mediante las interrelaciones que existen entre los miembros de la red. Los aprendizajes, según cuentan ellos, se inician desde edades muy tempranas hacia el interior de la familia. Es por esta razón que los miembros cabecera que conforman la red familiar constituyen, dentro de la propia familia, el motor impulsor de los procesos de formación en la transmisión del conocimiento tradicional y de los saberes populares, ya que este representa una herencia transmitida por sus antepasados a lo largo de los años. Dicha transmisión se realiza por vía oral y se corresponde con los códigos, vocabulario, hablas populares de la comunidad, lo que lo hace entendible y transmisible, al estar incorporados a la práctica cotidiana.

La relación individuo-institución aparece expresada en la interacción museo-portadores de conocimientos sobre la construcción de tecnologías y artes de pesca. Esta es una forma de que todos los involucrados adquieran conocimiento de esa lluvia de ideas que brota entre ellos mismos, lo cual hace que cada día se enriquezcan, se vuelvan más sabios y puedan seguir transmitiéndolo con precisión a las generaciones futuras.

La relación institución-institución se da fundamentalmente en museofamilia con el objetivo de preservar el portador y los contextos socioculturales donde se desarrollan y transforman las prácticas socioculturales. Esta relación es de gran importancia pues responde a los procesos de sistematización del patrimonio.

La relación familia-familia, garantiza los elementos de socialización, transmisión, empoderamiento de los saberes populares y portadores que facilitan el conocimiento y la praxis de la pesca de forma efectiva.

La relación de la familia con instituciones político-sociales, avala acciones de socialización, transmisión, empoderamiento, su 
sistematicidad y proceso de integración sociocultural a partir del conocimiento y divulgación de los diversos saberes.

Como puede apreciarse una vez más la práctica sociocultural se vuelve a colocar en el centro del proceso por el valor intrínseco de sistematización de la familia que, desde la perspectiva sociocultural se desenvuelve en complejos procesos de reproducción, donde la transmisión, la búsqueda de soluciones a los problemas y las inquebrantables interacciones dentro de la red familiar Devesa Herrera como colectivo. Estas juegan un papel importante, tanto en su interior como en la vecindad.

\section{Conclusiones}

- Las redes sociales de familia constituyen un subconjunto de las redes sociales, se forman a partir de lazos consanguíneos y sus vínculos se manifiestan en la cotidianidad.

- Las relaciones recíprocas que se establecen en las redes de familias marineras son fundamentales, pues cada red familiar desempeña un rol particular lo que incide favorablemente en beneficio de la comunidad.

- Los ancestros paternales de la red de familia Devesa Herrera son descendientes de familias pescadoras y proceden de España, específicamente de Islas Canarias.

- La red familiar Devesa Herrera garantiza la transmisión y resemantización de saberes, valores, símbolos y significados como expresión de la cotidianidad en la comunidad marinera Castillo-Perché a través de un sistema de relaciones y patrones de intercambio asociados a la actividad tecnoproductiva de pesca.

- La representación social de esta familia se encuentra en todos los proyectos que organiza y dirige la comunidad.

40 Ciencia y Sociedad 2013; 38(1): 27-46 
- La red familiar Devesa Herrera genera formas y expresiones identitarias propias, que se sustentan principalmente en la cooperación, la protección y la unidad familiar.

- Los vínculos con la actividad tecnoproductiva de la pesca son tan fuertes que la mayoría de los integrantes que conforman esta red familiar se dedican a tiempo completo a formar parte de su actividad económica fundamental.

- Los Devesa Herrera consideran que la familia representa el eje principal de sus relaciones y actividades porque a través de ella es que encuentran apoyo y seguridad.

- Los nexos entre esta red de familia son muy fuertes. Mantienen excelentes relaciones entre sí y se apoyan unos con otros constantemente en la búsqueda de soluciones a sus problemas.

- Esta familia no carece de nodo. En ella se observan dos tipos de acuerdo a sus fines: el nodo tecnoproductivo y el nodo festivo.

- Los vínculos afectivos son predominantes en los Devesa al girar constantemente a su alrededor y en todas las direcciones dentro de la propia red.

- Los tipos de relaciones manifestados por esta red familiar se dan principalmente entre individuo-individuo, individuo-grupo, los cuales se desarrollan dentro de los procesos de producción y reproducción de los conocimientos populares.

\section{Referencias bibliográficas}

Agüero, F. C. (2003). Antropología y currículo: una propuesta metodológica para el estudio de escuela secundaria del medio rural. En Moroto, F (2003). Trabajo realizado en el mismo programa de doctorado de la universidad de Oviedo y Cienfuegos. España: KRK. 
Albornoz, M. y Alfaraz, C. (2006). Redes de conocimiento: construcción, dinámica y gestión. Buenos Aires: (s. e.).

Álvarez, M. (1996). La familia cubana: cambios, actualidad y retos. Centro de Investigaciones Psicológicas y Sociales (CIPS), Ministerio de Ciencia, Tecnología y Medio Ambiente, La Habana: Ministerio de Ciencia, Tecnología y Medio Ambiente.

Banús, E. (2006). La Estrategia de redes de conocimiento adoptadas por UNESCO. En M. Albornoz, \& C. Alfaraz, Red de Conocimiento, construcción, dinámica y gestión (pp. 11-14). Buenos Aires: Oficina Regional de Ciencia para América Latina y el Caribe de la UNESCO.

Barcia, M. (2009). La familia: historia de su historia. [Monografía del Centro de Superación para la Cultura Juan Marinello]. Edición en formato digital.

Barrera, J. (1998). Generalidades sobre la familia en el Antiguo Régimen. Recuperado (s. f.) de: http://www.revistauniversidad.uson. mx.pdf.

Benítez Pérez, M. (2007). Panorama sociodemográfico de la familia cubana. La Habana: Editorial Félix Varela.

Díaz, E. (2005). Evaluación parcial del Proyecto Luna. La Habana: Editorial Universo Sur. Universidad de Cienfuegos.

Del Sol Delgado, B. (2011). La emigración santanderina: Su influencia en la región de Cienfuegos entre 1880-1920. Tesis en opción al título de máster. UCF por la Cienfuegos.

Denzin, N. y Lincoln, Y. (2004). Handbook of Qualitative Research. En Rodríguez Gómez, G., et al. Metodología de la investigación cualitativa. La Habana: Editorial Félix Varela.

Engels, F. (1975). El origen de la familia, la propiedad privada y el Estado. La Habana: Editorial de Ciencias Sociales. 
Espronceda Amor, M. E. (2009). Monografía del Centro de Superación para la Cultura Juan Marinello. Umbrales conceptuales para un enfoque antropológico de la familia: el parentesco. En fase de edición en formato digital.

Fernández, L. D.; Moros, J. L. (2004). Representaciones sociales en torno al trabajo, entre las familias de pescadores de las Islas de Zapara (Venezuela). Revista de Ciencias Humanas y Sociales, 44, pp. 138-155. Recuperado (s. f.) de http://www.subpesca.cl/ publicaciones/Pesca Artesanal.html

García, R. (2008). Los saberes populares en la comunidad del Castillo de Jagua. Un estudio de caso. Trabajo de diploma. UCF Cienfuegos.

González Gómez, C. I. y Basaldúa Hernández, M. (2007). La formación de redes sociales en el estudio de actores y familias. Perspectiva de estudio en historia y antropología. REDESRevista bispana para el análisis de redes sociales Vol. 12 (8), s. p. Recuperado (s. f.) de http://revista-redes.rediris.es/pdf-vol12/ Vol12_8.pdf.

Goyette, M. (2007). Redes sociales e inserción social: El interés de un enfoque relacional para la investigación en el trabajo social. Recuperado (s. f.) de http://www.revistatabularasa.org/ numerotres/goyette.pdf.

Granovetter, M. S. (1973). The Strength of Weak Ties, American Journal of Sociology, Vol. 78 (6), p. 1360.

Hage, J. y C. Alter. (2000). A typology of Interorganizational Relationships and Networks, en Hollingsworth, R. y Boyer, R. (eds.). Contemporary Capitalism. The 294 Embeddedness of Institutions, Cambridge: Cambridge University Press, pp. 94. 
Hernández García, M. (2010). La emigración vasca en Cienfuegos (1860-1898). [Tesis de opción para el título de máster por la UCF de Cienfuegos].

Hernández Sampieri, R. (2003). Metodología de la investigación social. La Habana: Editorial Félix Varela.

Legall, D. (1999). Aproximaciones sociológicas al estudio de la familia. Revista de Ciencias Humanas y Sociales. 6 (s. pp.). Recuperado (s. f.) de http://www.subpesca.cl/publicaciones/Pesca Artesanal.html

Merelo Guervós, J. J. (2004). Una introducción a las redes sociales. Recuperado (s. f.) de http://revista-redes.rediris.es/webredes/ talleres/redes-sociales.pdf.

Miranda Vera, C. E.; Castellanos González, M. E.; León Pérez, MSc. Ángel Raúl. (2008). Experiencias en el proceso de formación de redes del conocimiento. En Faloh Bejerano, R. \& Fernández de Alaiza, D. Redes de conocimiento. Conceptos y aplicaciones. La Habana: Academia.

Packman, M. (2002). Análisis de redes sociales: Conceptos fundamentales. Recuperado de http://www.buenastareas. com/ensayos/La-Importancia-De-Las-Redes.

Pérez González, E. (2010). Las normas de interacción sociocultural en el aprendizaje de la pesca artesanal en la familia Pérez Devesa en la comunidad marinera del Perché: un estudio de caso [Trabajo de diploma. UCF Cienfuegos].

Prats, J. (2004). Técnicas y recursos para la elaboración de tesis doctorales: bibliografía y orientaciones metodológicas. Barcelona: Universidad de Barcelona.

Rodríguez, F. (2002). La importancia de las redes sociales. Recuperado de http://revista-redes.rediris.es/webredes/ talleres/redes-sociales.pdf. 
Ritzel, G. (2006). Teoría sociológica contemporánea (tomo III). La Habana: Editorial Félix Varela.

Rodríguez, A.; Andrés, A. y Yulianov, E. (2003). Redes virtuales para la gestión del conocimiento: El caso de las universidades. Revista del Centro para la gestión del conocimiento en la universidad. (s. r.), p. 427-439.

Rodríguez Gómez, G. (2004). Metodología de la investigación cualitativa. La Habana: Editorial Félix Varela.

Royero, J. (2001). Las redes sociales de conocimiento: El nuevo reto de las organizaciones de investigación científica y tecnológica. Recuperado de www.monografias.com.

Sánchez Seijó, Y. (2010). El estudio de las prácticas tecnoproductivas en la familia marinera Vera Sánchez de la comunidad Castillo de Jagua. Estudio de caso. [Trabajo de diploma por UCF de Cienfuegos].

Shannin, T. (1983). La clase incómoda. España: Alianza Editoriales.

Soler Marchán, D. (2006). Informe de investigación al catálogo sobre la comunidady bebidas marineras en Cienfuegos, Cienfuegos: CPPC.

Soler Marchán, D. (2009). Prácticas tecnoproductiva. Metodología Utilitaria. Encuentro Internacional de Capacitación Cuba y Ecuador. Cienfuegos, Cuba.

Taylor, S. J. y Bogdan, R. (1987). Introducción a los métodos cualitativos de investigación. Barcelona: Ediciones Paidós Ibérica, S. A.

Vargas, J. (2003). Teoría de la acción colectiva: Sociedad civil y movimientos sociales en las nuevas formas de gobernabilidad en Latinoamérica. Revista Nómadas, N. 7 (s. pp.). 
Vera, A. (2009). Monografía del Centro de Superación para la Cultura Juan Marinello: El qué y el para qué de la historia de la familia. [En fase de edición en formato digital].

\section{Cynthia Acevedo Rodríguez}

Tiene una licenciatura en Estudios Socioculturales por la Universidad de Cienfuegos, Cuba. Se dedica a la investigación social; ha publicado varios trabajos de carácter etnográfico.

\section{Nereyda Moya Padilla}

Es profesora Titular del Departamento de Ciencias Sociales de la Universidad de Cienfuegos, Cuba. Ha participado y publicado diversos trabajos sobre el concepto de lo sociocultural, la mediación de la tradición tecno-productiva en el entorno regional, entre otros temas actuales. Participó e intervino en la III Conferencia Internacional sobre La obra de Carlos Marx y los desafíos del siglo XXI.

Email: nmoyapadilla@gmail.com 